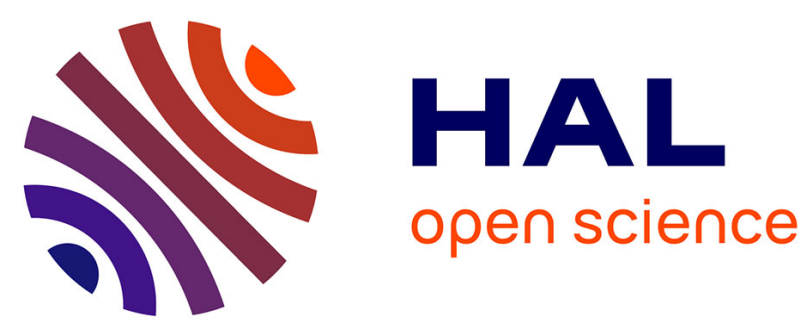

\title{
Silicon made resonant microcantilever: dependence of the chemical sensing performances on the sensitive coating thickness
}

Frédéric Lochon, Ludivine Fadel, Isabelle Dufour, Dominique Rebiere, Jacques Pistré

\section{To cite this version:}

Frédéric Lochon, Ludivine Fadel, Isabelle Dufour, Dominique Rebiere, Jacques Pistré. Silicon made resonant microcantilever: dependence of the chemical sensing performances on the sensitive coating thickness. Materials Science \& Engineering C, 2006, 26 (2-3), pp.348-353. hal-00203739

\section{HAL Id: hal-00203739 \\ https://hal.science/hal-00203739}

Submitted on 13 Nov 2014

HAL is a multi-disciplinary open access archive for the deposit and dissemination of scientific research documents, whether they are published or not. The documents may come from teaching and research institutions in France or abroad, or from public or private research centers.
L'archive ouverte pluridisciplinaire $\mathbf{H A L}$, est destinée au dépôt et à la diffusion de documents scientifiques de niveau recherche, publiés ou non, émanant des établissements d'enseignement et de recherche français ou étrangers, des laboratoires publics ou privés. 
Preprint - Materials Science \& Engineering C, Vol 26/2-3, 2006, pp. 348-353. (doi:

10.1016/j.msec.2005.10.079)

\section{SILICON MADE RESONANT MICROCANTILEVER: DEPENDENCE OF THE CHEMICAL SENSING PERFORMANCES ON THE SENSITIVE COATING THICKNESS}

Frédéric Lochon*, Ludivine Fadel, Isabelle Dufour, Dominique Rebière, Jacques Pistré Laboratoire IXL - CNRS UMR 5818 - ENSEIRB / Université Bordeaux 1, 351 cours de la Libération, 33405 Talence Cedex, France

Email: lochon@ixl.fr

\section{ABSTRACT}

A gas sensor based on the use of a resonating microcantilever has been realized by using a polymer sensitive coating. From the theoretical study of the microcantilever sensitivity, it has been deduced that the sensitivity is enhanced when the resonant frequency or the sensitive coating thickness are increased. The sensitive coating thickness influence has then been verified experimentally by using PEUT (polyetherurethane) as sensitive coating for ethanol detection. From these measurements, some drawbacks are shown: the coating thickness increase leads to a sensor response time increase and a frequency noise increase which worsens the limit of detection. Conclusions are then made about the sensitive coating optimization depending on application constraint considerations.

\section{KEYWORDS}

Microcantilever sensor, resonant frequency, sensitive layer thickness

\section{Introduction}

Resonant microcantilevers are used for more than 10 years as chemical sensors [1-10]. Indeed, because microcantilevers are very sensitive mass sensors, it is possible to detect small variations of sensitive layer mass by depositing a sensitive layer at the microcantilever surface. The sorption of gas molecules into the sensitive coating makes then possible their detection. Thus, by knowing the sensitivity of the microcantilever, it is possible to estimate the gas concentration by measuring the resonant frequency shift. 
Preprint - Materials Science \& Engineering C, Vol 26/2-3, 2006, pp. 348-353. (doi:

10.1016/j.msec.2005.10.079)

This paper presents some results obtained with our special shaped microcantilevers combined with a polymer sensitive coating for ethanol detection. The influence of the sensitive coating thickness is detailed theoretically and confirmed by the presented experiments.

\section{Used microcantilevers}

In opposition to classical parallelepiped-shaped microcantilevers, this paper presents results obtained with non-parallelepiped-shaped microcantilevers combined with a PEUT (polyetherurethane) sensitive layer (Figure 1).

The microcantilevers are composed of two parts: the arm and the plate. The arm corresponds to the part which links the substrate to the plate. For sensitivity reasons, all the realized microcantilevers have a plate larger than the arm, as the modification of resonant frequency is more important when a mass is added at the microcantilever free-end.
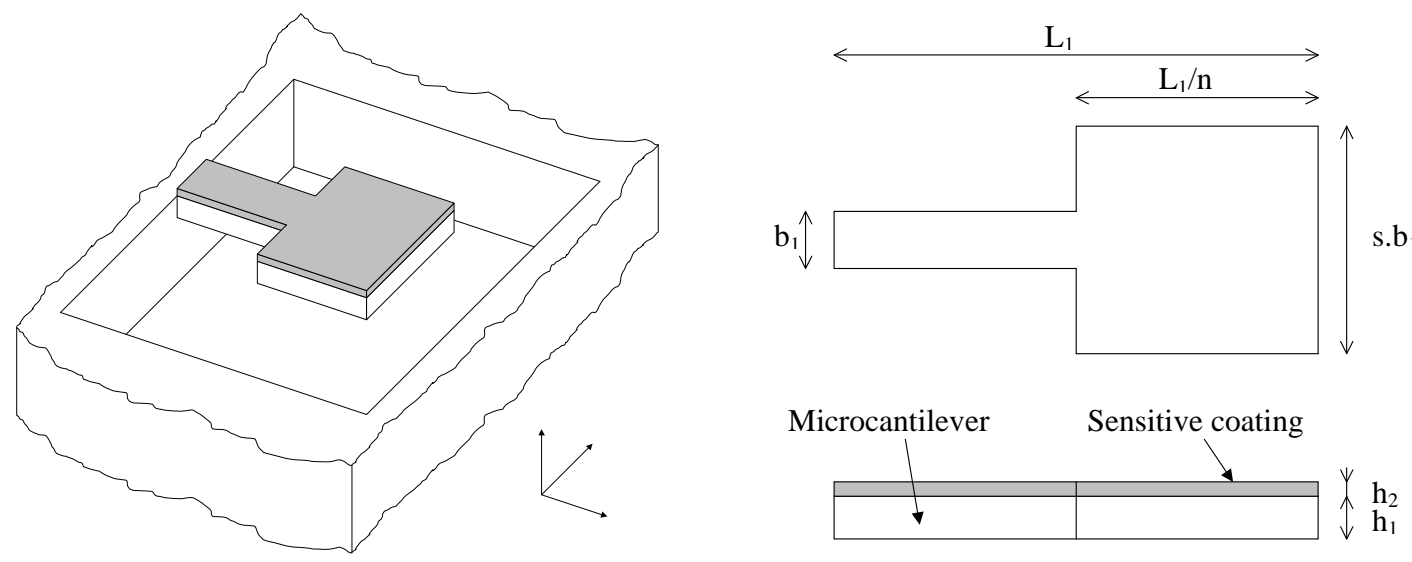

Figure 1: A microcantilever and its sensitive coating

The studied microcantilever dimensions are:

- $\mathrm{L}_{1}=1.5 \mathrm{~mm}, \mathrm{n}=1.5, \mathrm{~b}_{1}=200 \mu \mathrm{m}, \mathrm{s}=5, \mathrm{~h}_{1}=85 \mu \mathrm{m}$ (resonating at $22.770 \mathrm{kHz}$ ).

- $\mathrm{L}_{1}=2 \mathrm{~mm}, \mathrm{n}=2, \mathrm{~b}_{1}=400 \mu \mathrm{m}, \mathrm{s}=2.5, \mathrm{~h}_{1}=89 \mu \mathrm{m}$ (resonating at $17.780 \mathrm{kHz}$ ).

\section{Modeling}

As it will be shown later in the paper, the resonant frequency is a key parameter to design a sensor with a good sensitivity, since the sensitivity of resonant microcantilever sensors is directly proportional to the resonant frequency (section 3.2). 
Preprint - Materials Science \& Engineering C, Vol 26/2-3, 2006, pp. 348-353. (doi: 10.1016/j.msec.2005.10.079)

\section{Resonant frequency without coating}

By using the Rayleigh's method which consists in equaling the kinetic energy and the potential energy, a good approximation of the resonant frequency can be calculated by knowing a realistic expression of the microcantilever deflection.

It is then possible to express the approximate resonant frequency of an homogeneous nonparallelepiped shaped microcantilever [11]:

$$
f=\frac{h_{1}}{2 \pi L_{1}^{2}} \sqrt{\frac{\hat{E}_{1}}{\xi_{1} \rho_{1}}}
$$

where:

$\xi_{1}$ is a rational fraction of $s$ and $n$ ( $n$ is the ratio of the total length of the structure to the plate length, and $s$ is the ratio of the plate width to the arm width, see Figure 1), $\rho_{1}$ the microcantilever mass density, $\hat{E}_{1}$ the microcantilever effective Young modulus, $h_{1}$ the thickness, and $L_{1}$ the length of the microcantilever.

The expressions found by using the Rayleigh's method are valid for a microcantilever resonating in vacuum. In our case, since the microcantilever resonates in air, a slight modification of the resonant frequency appears due to losses and fluid inertia.

This modification is usually negligible because the quality factor (linked to the losses) of the used microcantilevers is relatively high (at least better than 100).

\section{Resonant frequency with coating}

\subsection{Analytical expression}

When a layer is deposited on top of a microcantilever, its resonant frequency is modified for two reasons: the added mass tends to decrease the frequency (see section 2.2) while the Young modulus of the layer tends to increase the frequency (see section 2.3).

Using the Rayleigh's method, the microcantilever resonant frequency with a sensitive coating deposited can be expressed [12]:

$$
f=\frac{1}{2 \pi L_{1}^{2}} \sqrt{\frac{1}{\xi_{1}\left(\rho_{1} h_{1}+\rho_{2} h_{2}\right)}} \sqrt{\frac{h_{1}^{4} \hat{E}_{1}^{2}+h_{2}^{4} \hat{E}_{2}^{2}+2 h_{1} h_{2} \hat{E}_{1} \hat{E}_{2}\left(2 h_{1}^{2}+3 h_{1} h_{2}+2 h_{2}^{2}\right)}{h_{1} \hat{E}_{1}+h_{2} \hat{E}_{2}}}
$$


Preprint - Materials Science \& Engineering C, Vol 26/2-3, 2006, pp. 348-353. (doi:

10.1016/j.msec.2005.10.079)

where $\rho_{2}$ is the deposited layer mass density, $h_{2}$ is the deposited layer thickness and $\hat{E}_{2}$ is the deposited layer effective Young modulus.

From the resonant frequency expression (2), two terms can be extracted:

- The mass effect term:

$$
G=\sqrt{\frac{1}{\rho_{1} h_{1}+\rho_{2} h_{2}}}
$$

- The Young modulus dependent term:

$$
Y=\sqrt{\frac{h_{1}^{4} \hat{E}_{1}^{2}+h_{2}^{4} \hat{E}_{2}^{2}+2 h_{1} h_{2} \hat{E}_{1} \hat{E}_{2}\left(2 h_{1}^{2}+3 h_{1} h_{2}+2 h_{2}^{2}\right)}{h_{1} \hat{E}_{1}+h_{2} \hat{E}_{2}}}
$$

\subsection{Mass effect}

The term $G$ clearly shows the influence of the material masses: when the mass densities increase, the resonant frequency decreases or when the thicknesses increase (i.e. the mass increases), the resonant frequency decreases.

Thus, using light materials will allow high resonant frequencies and an increased sensitivity (see section 3.2).

\subsection{Young modulus effect}

The term $Y$ can also be expressed:

$$
Y=\sqrt{\hat{E}_{1} h_{1}^{3}} \sqrt{\frac{1+\alpha \beta\left(4+6 \beta+4 \beta^{2}+\alpha \beta^{3}\right)}{1+\alpha \beta}}
$$

With $\alpha=\frac{\hat{E}_{2}}{\hat{E}_{1}}$ and $\beta=\frac{h_{2}}{h_{1}}$.

From equation (5), it can be seen that $Y$ has a minimum value of $Y_{0}=\sqrt{\hat{E}_{1} h_{1}^{3}}$ which is obtained when the sensitive coating is very thin $\left(h_{2} \rightarrow 0\right)$ or when its Young modulus is very $\operatorname{low}\left(\hat{E}_{2} \rightarrow 0\right)$.

If the sensitive coating has a non negligible Young modulus, then the resonant frequency tends to be increased when the sensitive coating thickness increases. 
Preprint - Materials Science \& Engineering C, Vol 26/2-3, 2006, pp. 348-353. (doi:

10.1016/j.msec.2005.10.079)

\section{Gas sensor response}

\subsection{Gas sorption}

The sorption of gas molecules into the sensitive coating is expressed as a modification of the coating mass density. This modification depends on the affinity of the coating for the adsorbed gas and on the gas concentration in the surrounding medium.

The modified mass density of the coating is expressed:

$$
\rho_{2}\left(C_{g}\right)=\rho_{2}+K C_{g}
$$

where $\rho_{2}$ is the mass density of the coating without adsorbed molecules, $K$ is the partition coefficient (defined as the ratio between the gas concentration in the coating and the gas concentration in the surrounding medium) and $C_{g}$ is the gas concentration in the surrounding medium.

The resulting resonant frequency is then expressed:

$$
f=\frac{h_{1}}{2 \pi L_{1}^{2}} \sqrt{\frac{\hat{E}_{1}}{\xi_{1}\left(\rho_{1}+\rho_{2} \beta+K \beta C_{g}\right)}} \sqrt{\frac{1+\alpha \beta\left(4+6 \beta+4 \beta^{2}+\alpha \beta^{3}\right)}{1+\alpha \beta}}
$$

\subsection{Gas sensitivity}

For small variations of the resonant frequency, the gas sensitivity $S_{C_{g}}$ is defined as:

$$
S_{C_{g}}=\left|\left[\frac{\partial f}{\partial C_{g}}\right]_{C_{g}=0}\right|
$$

Then, substituting (7) in (8) gives:

$$
S_{C_{g}}=\frac{K h_{2}}{2\left(\rho_{1} h_{1}+\rho_{2} h_{2}\right)}[f]_{C_{g}=0}
$$

Using (9) and (7), it is possible to demonstrate that a theoretical optimum coating thickness exists for very low sensitive coating Young modulus [13]. This optimal thickness can be expressed as a function of the cantilever thickness and the mass densities of the cantilever and coating: 
Preprint - Materials Science \& Engineering C, Vol 26/2-3, 2006, pp. 348-353. (doi: 10.1016/j.msec.2005.10.079)

$$
\rho_{2} h_{2}=2 \rho_{1} h_{1}
$$

For higher sensitive coating Young modulus, no optimal coating thickness exists since the sensitivity is always increased when increasing the sensitive coating thickness.

\section{Experimental results}

\section{Test bench}

The actuation of the microcantilever at its resonant frequency is done with an electronic device which permits the microcantilever oscillation. This frequency is then measured by an HP53131 (frequency counter) and sent to a PC (Figure 2).

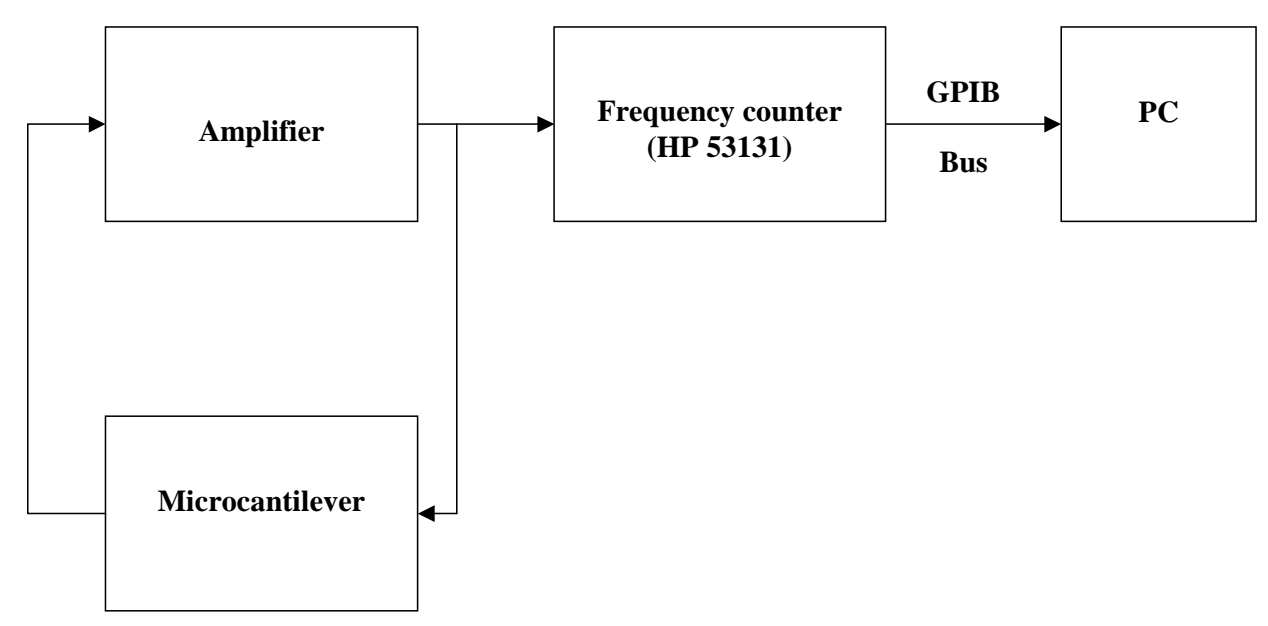

Figure 2: Microcantilevers resonant frequency measurement setup

\section{Mass effect}

The modeling process about mass effect has been verified by depositing the sensitive layer on the microcantilever top surface. Because the chosen sensitive material (PEUT) has a very low Young modulus ( $E_{2}=6.9 \mathrm{MPa}$ ) compared to the cantilever material (silicon $E_{1}=130 G P a$ ), the ratio sensitive coating Young modulus to cantilever material Young modulus is very low $(\alpha<5 e-5)$. Thus, the sensitive coating Young modulus cannot modify the resonant frequency and thus any modification of the resonant frequency is only due to an added mass. To verify the dependence of the resonant frequency upon an added mass, the resonant frequency of a cantilever has been measured in oscillator configuration (Figure 2) during the sensitive coating deposition. 
Preprint - Materials Science \& Engineering C, Vol 26/2-3, 2006, pp. 348-353. (doi: 10.1016/j.msec.2005.10.079)

The deposition is performed with a spray coating device which allows reproducible and uniform sprays. The spray coater, which has been characterized, deposits a constant sensitive coating thickness per pulverization depending on multiple parameters (viscosity of the solution, pulverization pressure, ...). Thus, the deposited sensitive coating thickness has to be determined a posteriori using (2) with $E_{2}<<E_{1}$ :

$$
h_{2}=\frac{\rho_{1} h_{1}}{\rho_{2}}\left(\frac{f_{0}^{2}}{f^{2}}-1\right)
$$

where $f_{0}$ is the resonant frequency without coating.

Figure 3 shows the modification of the resonant frequency during the spray and the estimated deposited coating thickness using (11):

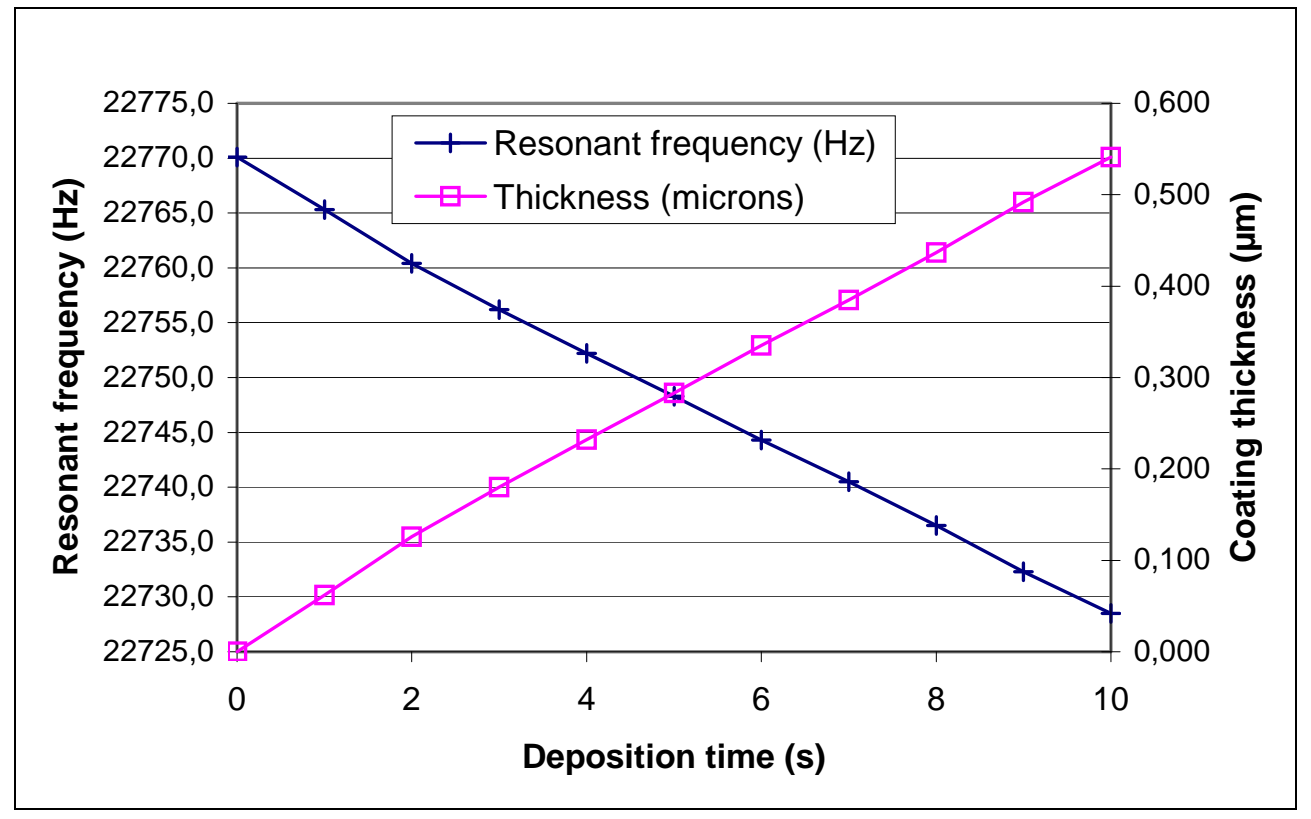

Figure 3: Resonant frequency and estimated coating thickness versus deposition time

\section{Gas detection}

To verify the sensitivity of the sensor, a microcantilever with its associated sensitive coating is inserted in a gas-line and is exposed to different ethanol concentrations (Figure 4). 
Preprint - Materials Science \& Engineering C, Vol 26/2-3, 2006, pp. 348-353. (doi:

10.1016/j.msec.2005.10.079)

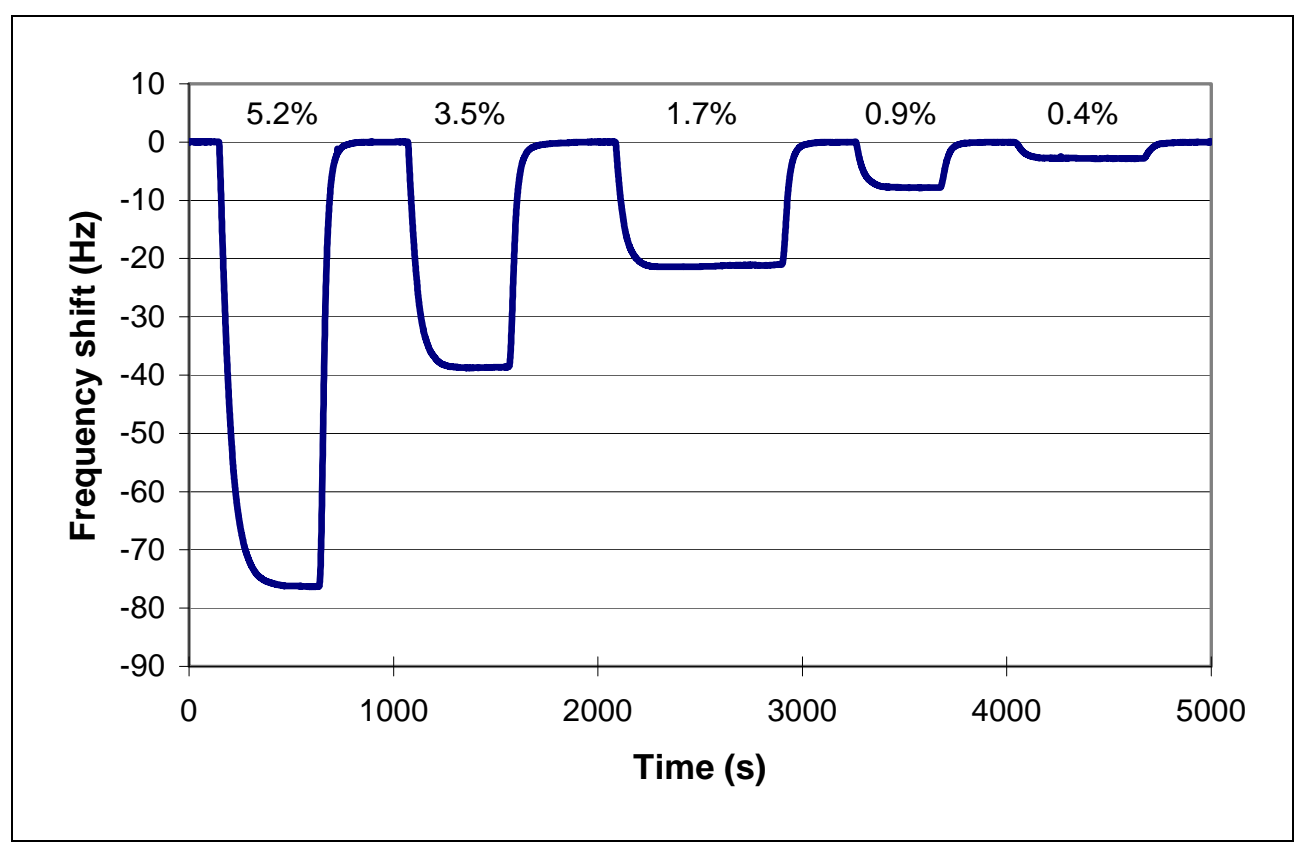

Figure 4: Frequency shift (drift removed) for a microcantilever resonating at $16.9 \mathrm{kHz}$ with a sensitive coating thickness of $21 \mu \mathrm{m}$, exposed to ethanol at different concentrations

Then, different coating thicknesses have been used to show the thickness influence upon the sensitivity, the response time and the limit of detection.

As shown in Figure 5 and as predicted theoretically, the sensitivity increases when the coating thickness increases.

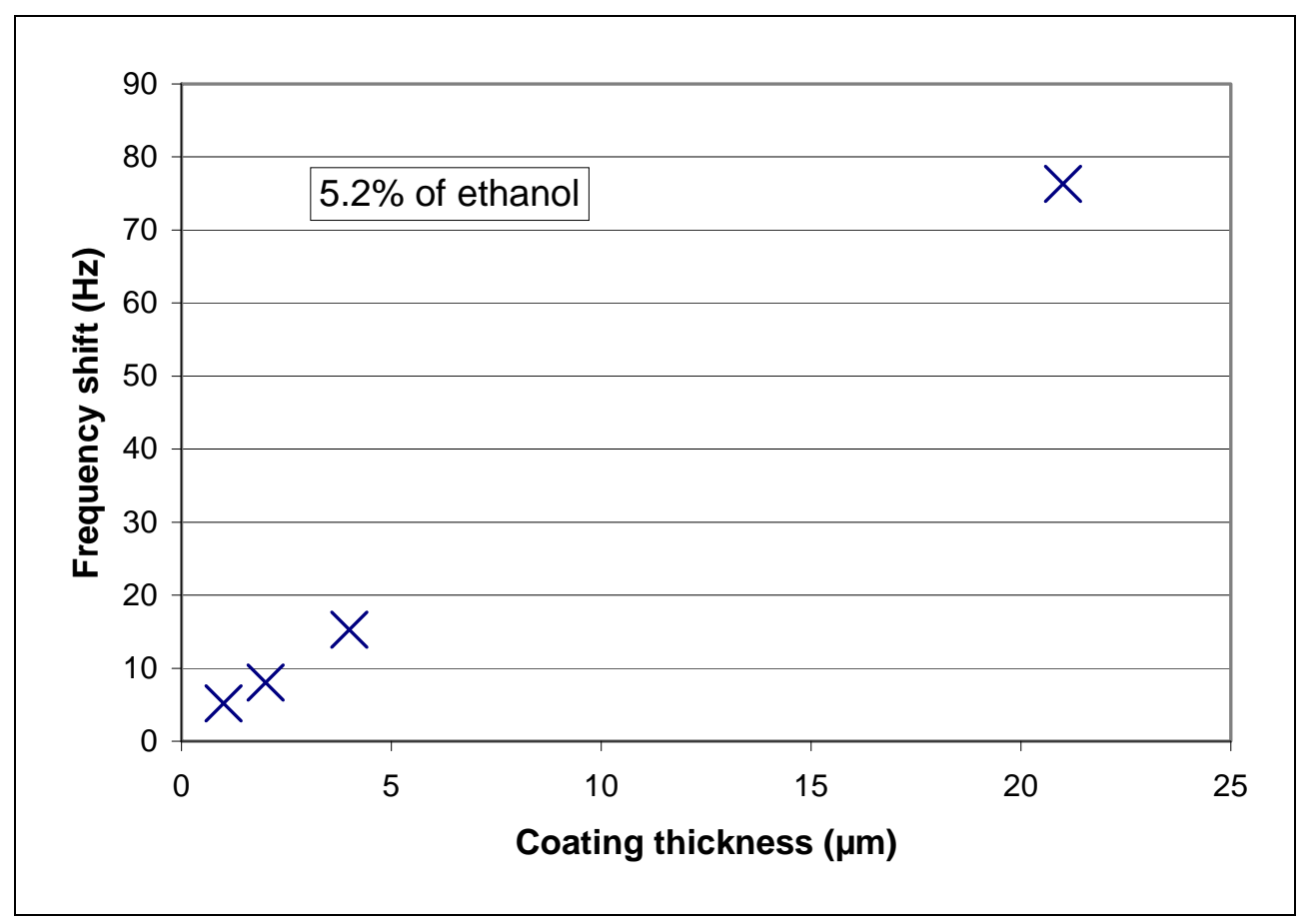

Figure 5: Frequency shift (absolute value) for different coating thickesses for a microcantilever exposed to 5.2\% of ethanol 
Preprint - Materials Science \& Engineering C, Vol 26/2-3, 2006, pp. 348-353. (doi: 10.1016/j.msec.2005.10.079)

In order to compare these measurements with the theoretical expression (9), the measured normalized frequency shift and theoretical normalized frequency shift, using the partition coefficient of the literature (K=950) [14], have been plotted (Figure 6).

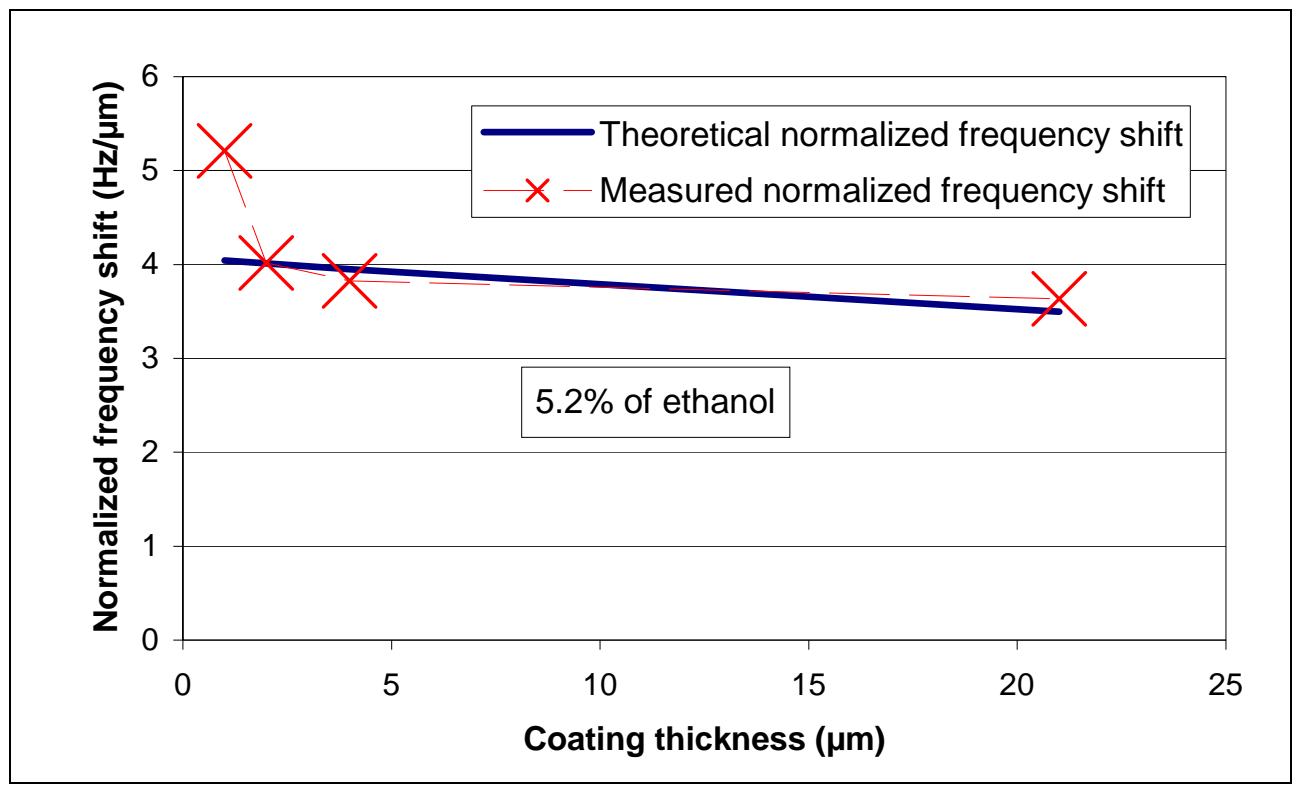

Figure 6: Normalized frequency shift (ratio frequency shift to coating thickness) versus coating thickness for a microcantilever exposed to $5.2 \%$ of ethanol

The measurements are in good adequacy with the theory excepted for the first point $(1 \mu \mathrm{m})$.

The reason of the discrepancy for the first point has not been clearly identified but a surface interaction between the gas and the coating may explain this phenomenon: since the area in contact with the gas is approximately the same whatever the coating thickness is, a gas quantity trapped close to the surface would increase the sensitivity for thin sensitive coatings.

Table 1 shows the partition coefficient which is calculated from (9) using the estimated coating thickness, the measured resonant frequency with the deposited coating and the measured frequency shift for an exposition to $5.2 \%$ of ethanol.

The obtained values are in good accordance to the literature excepted when the coating is $1 \mu \mathrm{m}$ thick. This difference may be explained by surface interactions as said previously. 
Preprint - Materials Science \& Engineering C, Vol 26/2-3, 2006, pp. 348-353. (doi: 10.1016/j.msec.2005.10.079)

Table 1: Deduced partition coefficient for each coating thicknesses

\begin{tabular}{|c|c|c|c|}
\hline $\begin{array}{c}\text { Coating } \\
\text { thickness }(\mu \mathrm{m})\end{array}$ & $\begin{array}{c}\text { Frequency shift } \\
(\mathrm{Hz})\end{array}$ & $\begin{array}{c}\text { Resonant } \\
\text { frequency }(\mathrm{Hz})\end{array}$ & $\begin{array}{c}\text { Deduced partition coefficient } \\
\text { (as defined in section III.3.1) }\end{array}$ \\
\hline 1 & -5.21 & 17758 & 1220 \\
\hline 2 & -8.03 & 17699 & 951 \\
\hline 4 & -15.3 & 17614 & 987 \\
\hline 21 & -76.3 & 16907 & \\
\hline
\end{tabular}

\section{Response time}

The responses of cantilevers exposed to $5.2 \%$ of ethanol with different coating thicknesses have been normalized and are presented in Figure 7. From this figure, one thing can be noticed: the response time is approximately the same for thin coatings and is bigger for the 21 $\mu \mathrm{m}$ thick coating.

This can be explained by the response time of the chamber. Indeed, the chamber is, naturally, filled progressively and its response time has to be taken into account. If the chamber response time is greater than the sensor response time, then the observed response time is mainly due to the chamber.

In fact, in our case for coating thicknesses up to $4 \mu \mathrm{m}$, the observed response time is due to the chamber while for the $21 \mu \mathrm{m}$ thick coating the adsorption time affect the observed response time. 
Preprint - Materials Science \& Engineering C, Vol 26/2-3, 2006, pp. 348-353. (doi: 10.1016/j.msec.2005.10.079)

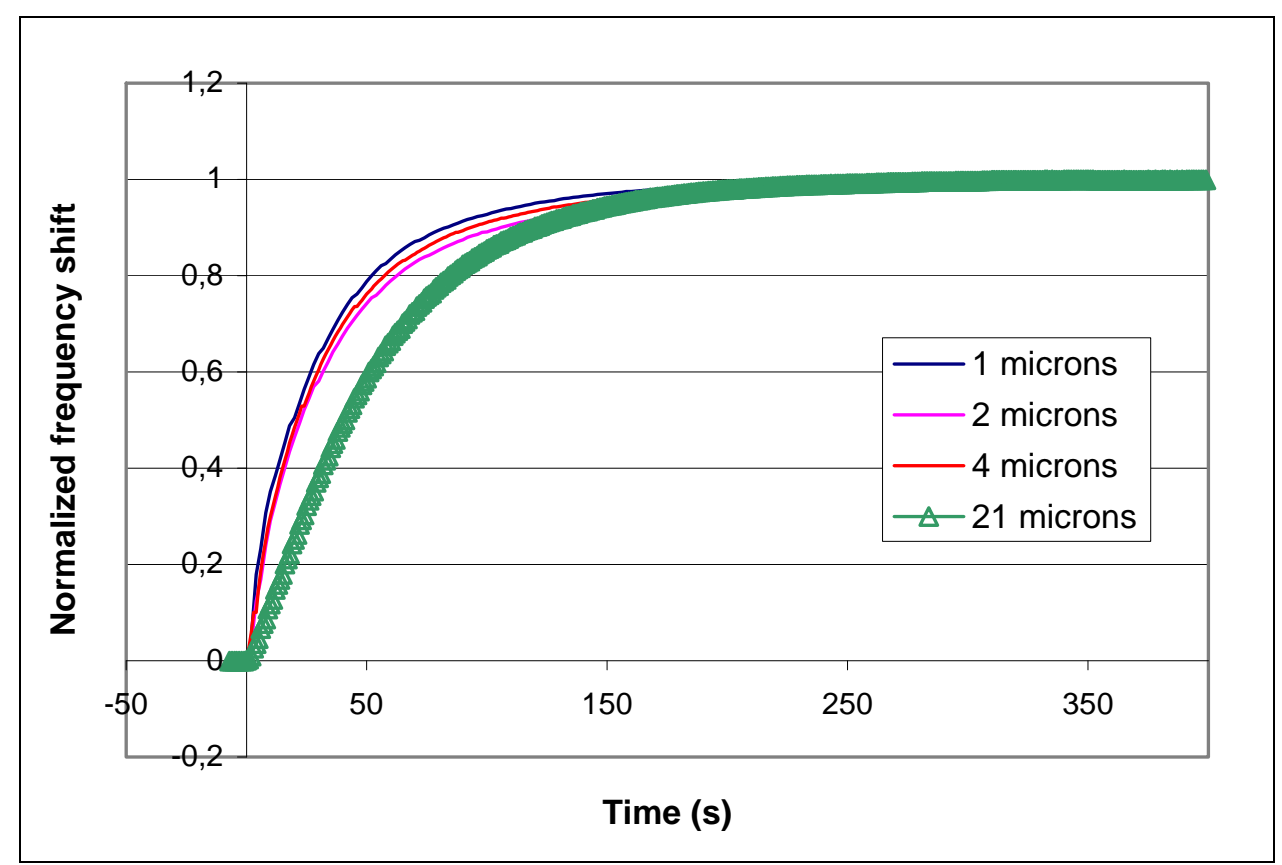

Figure 7: Normalized frequency shift versus time for different coating thicknesses

The increased response time for the $21 \mu \mathrm{m}$ thick coating clearly shows that the increase in sensitivity (Figure 5) can be achieved to the detriment of the response time (Figure 7).

\section{Limit of detection}

The limit of detection (L.O.D.), calculated from the measured sensor sensitivity $S_{C_{g}}$ and the measured frequency noise $\Delta f_{\text {noise }}$, is represented in Table 2:

L.O.D. $=\frac{3 \Delta f_{\text {noise }}}{S_{C_{g}}}$

It can be seen that, the frequency noise and the sensitivity increase when the sensitive coating thickness increases. Because the frequency noise does not increase in the same way as the sensitivity, the limit of detection is minimal, and thus optimal, for a sensitive coating thickness between $1 \mu \mathrm{m}$ and $21 \mu \mathrm{m}$. 
Preprint - Materials Science \& Engineering C, Vol 26/2-3, 2006, pp. 348-353. (doi: 10.1016/j.msec.2005.10.079)

Table 2: Limit of detection of ethanol for a microcantilever resonating at $17.7 \mathrm{kHz}$

\begin{tabular}{|c|c|c|c|}
\hline Sensitive coating thickness & $1 \mu \mathrm{m}$ & $4 \mu \mathrm{m}$ & $21 \mu \mathrm{m}$ \\
\hline Sensitivity (mHz/ppm) & 0.1 & 0.29 & 1.5 \\
\hline Frequency noise (Hz) & 0.001 & 0.002 & 0.015 \\
\hline Estimated limit of detection (ppm) & 30 & 20.4 & 30 \\
\hline
\end{tabular}

The existence of an optimum coating thickness also demonstrates that the coating thickness has to be chosen carefully to avoid large response times and to allow an optimized sensor response.

\section{Conclusion}

The sensitive coating influence upon sensitivity, response time and limit of detection has been shown and the presence of an optimal sensitive coating thickness has been observed.

The sensor, using a $4 \mu \mathrm{m}$ thick PEUT sensitive coating, has been able to detect ethanol with a sensitivity of about $0.29 \mathrm{mHz} / \mathrm{ppm}$ with a frequency noise of about $2 \mathrm{mHz}$.

The selectivity of the device has not been studied, but similar results could also be achieved when detecting other gases since the PEUT is sensitive to most volatile organic compounds (VOCs).

From the measurements, conclusions have been done about the sensitive coating influence upon sensitivity, response time and limit of detection. Theses conclusions raise issues about the optimization of the sensitive coating thickness.

Because the determination of the exact value of the optimal sensitive coating thickness would require much more experimental work, it could be interesting to characterize precisely the frequency noise. This characterization requires the theoretical determination of the viscoelastic losses that occurs in the sensitive coating.

More generally, a theoretical study on the losses in resonant microcantilever sensors will significantly help the designers in reducing the frequency noise and thus adapting the microcantilever geometries and enhancing the limit of detection. 
Preprint - Materials Science \& Engineering C, Vol 26/2-3, 2006, pp. 348-353. (doi: 10.1016/j.msec.2005.10.079)

\section{ACKNOWLEDGEMENTS}

This work was sponsored in part by the French Agency for Environment and Energy Management (ADEME) and the "Région Aquitaine".

The authors wish to thank Jean-Luc Lachaud and Serge Destor for their help in the design of the spray coating device, and Olivier Français and Patrick Sangouard for the microcantilever fabrication.

\section{REFERENCES}

[1] D. Lange, C. Hagleitner, A. Hierlemann, O. Brand and H. Baltes, Complementary metal oxide semiconductor cantilever arrays on a single chip: mass-sensitive detection of volatile organic compounds, Analytical Chemistry, 74 (2002), pp.3084-3095.

[2] T.A. Betts, C.A. Tipple, M.J. Sepaniak and P.G. Datskos, Selectivity of chemical sensors based on micro-cantilevers coated with thin polymer films, Analytica Chimica Acta 422 (2000), pp.89-99.

[3] F.M. Battiston, J.-P. Ramseyer, H.P. Lang, M.K. Baller, Ch. Gerber, J.K. Gimzewski, E. Meyer and H.-J. Güntherodt, A chemical sensor based on a microfabricated cantilever array with simultaneous resonance-frequency and bending read-out, Sensors and Actuators B, 77 (2001), pp.122-131.

[4] M. Sepaniak, P. Datskos, N. Lavrik and C. Tipple, Microcantilever transducers: a new approach in sensor technology, Analytical Chemistry, A-Pages, 74 (21) (2002) pp.568A-575A

[5] P.G. Datskos, T. Thundat, N.V. Lavrik, Micro and nanocantilever sensors, Encyclopedia of nanoscience and nanotechnology, Edited by H.S. Nalwa (2004) 10 pages.

[6] J. Thaysen, A.D. Yalcinkaya, P. Vettiger and A. Menon, Polymer-based stress sensor with integrated readout, Journal of Physics D: applied Physics, 35 (2002) pp. 2698-2703.

[7] J. Zhou, P. Li, S. Zhang, Y. Long, F. Zhou, Y. Huang, P. Yang and M. Bao, Zeolitemodified microcantilever gas sensor for indoor air quality control, Sensors and Actuators B, 94 (2003) pp.337-342.

[8] G. Abadal, Z.J. Davis, B. Helbo, X. Borrisé, R. Ruiz, A. Boisen, F. Campabadal, J. Esteve, E. Figueras, F. Perez-Murano and N. Barniol, Electromechanical model of a resonating nano-cantilever-based sensor for high-resolution and high-sensitivity mass detection, Nanotechnology 12 (2001) pp. 100-104. 
Preprint - Materials Science \& Engineering C, Vol 26/2-3, 2006, pp. 348-353. (doi: 10.1016/j.msec.2005.10.079)

[9] R. Raiteri, M. Grattarola, H.J. Butt and P. Skadal, Micromechanical cantilever-based biosensors, Sensors and Actuators B, 79 (2001) pp.115-126.

[10] R. Berger, H.P. Lang, J.P. Ramseyer, F. Battiston, J.H. Fabian, L. Scandella, C. Andreoli, J. Brugger, M. Despont, P. Vettiger, E. Meyer, H.J. Güntherodt, C. Gerber and J.K. Gimzewski, Transduction principles and integration of chemical sensors into a micromechanical array device, Sensor Technology in the Netherlands: State of the art, ed. A. van den Berg and P. Bergveld (Kluwer, Dordrecht, 1998) pp. 33-??.

[11]I. Dufour and L. Fadel, Resonant microcantilever type chemical sensors: analytical modeling in view of optimization, Sensors and Actuators B, 91 (2003) pp.353-361.

[12] S. P. Timoshenko, Strength of materials, D. Van Nostran Company, Princeton, 1941.

[13]F. Lochon, I. Dufour and D. Rebière, An alternative solution to improve sensitivity of resonant microcantilever chemical sensors: comparison between using high-order modes and reducing dimensions, Sensors and Actuators B, 2005 (accepted).

[14]D. Lange, O. Brand and H. Baltes, CMOS cantilever sensor systems Atomic-ForceMicroscopy and Gas Sensing Applications (Berlin: Springer) 142 pp, 2002 